\title{
Fixed Points Associated to Power of Normal Completely Positive Maps*
}

\author{
Haiyan Zhang, Hongying Si \\ College of Mathematics and Information Science, Shangqiu Normal University, Shangqiu, China \\ Email: csqam@163.com
}

Received 1 April 2016; accepted 21 May 2016; published 24 May 2016

Copyright (C) 2016 by authors and Scientific Research Publishing Inc.

This work is licensed under the Creative Commons Attribution International License (CC BY).

http://creativecommons.org/licenses/by/4.0/

c) (i) Open Access

\section{Abstract}

Let $\varphi_{\mathrm{A}}$ be a normal completely positive map with Kraus operators $\mathrm{A}=\left\{E_{k}\right\}_{k=1}^{n}$. An operator $X$ is said to be a fixed point of $\varphi_{\mathrm{A}}$, if $\varphi_{\mathrm{A}}(X)=X$. Let $B(H)^{\varphi_{\mathrm{A}}}$ be the fixed points set of $\varphi_{\mathrm{A}}$. In this paper, fixed points of $\varphi_{\mathrm{A}}^{j}$ are considered for $1<j<+\infty$, where $\varphi_{\mathrm{A}}^{j}$ means $j$-power of $\varphi_{\mathrm{A}}$. We obtain that $B(H)^{\varphi_{A}^{2 j}}=B(H)^{\varphi_{A}}+B(H)^{-\varphi_{A}}$ and $B(H)^{\varphi_{A}^{2 j-1}}=B(H)^{\varphi_{A}}$ for integral $j>1$ when $A$ is self-adjoint and commutable. Moreover, $B(H)^{\varphi_{\mathrm{A}}^{j}}=B(H)^{\varphi_{\mathrm{A}}}$ holds under certain condition.

\section{Keywords}

\section{Fixed Point, Power, Completely Positive Map}

\section{Introduction}

Completely positive maps are founded to be very important in operator algebras and quantum information. Especially recent years, it has a great development since a quantum channel can be represented by a trace preserving completely positive map. Fixed points of completely positive map are useful in theory of quantum error correction and quantum measurement theory and have been studied in several papers from different aspects, many interesting results have been obtained (see [1]-[12]).

For the convenience of description, let $H$ be a separable complex Hilbert space and $B(H)$ be the set of all bounded linear operators on $H$. Let $\Phi$ on $B(H)$ be a contractive $(\|\Phi\|)$ map. As we know, every contractive and normal (or weak ${ }^{*}$ continuous) completely positive map $\Phi$ on $B(H)$ is determined by a row contraction on $H$ in the sense that

*Fixed points of completely positive maps. 


$$
\Phi(X)=\sum_{k=1}^{n} E_{k} X E_{k}^{*}, \forall X \in B(H)
$$

where if $n=+\infty$, the convergence is in the weak * topology (see [13] and [14]) and then denoting $\varphi_{\mathrm{A}}=\Phi$, we call $\varphi_{\mathrm{A}}$ a completely positive map associated with A.

Let $\mathrm{A}=\left\{E_{k}\right\}_{k=1}^{n}$ be an at most countable subset of $B(H)$ with $\sum_{k=1}^{n} E_{k} E_{k}^{*} \leq I$, where the series is convergent in the strong operator topology. In this case, $\mathrm{A}$ is called a row contraction. Then $\varphi_{\mathrm{A}}(X)=\sum_{k=1}^{n} E_{k} X E_{k}^{*}$ is well defined on $B(H)$ and also a normal completely positive map. Moreover, we denote $j$-power for $1<j<+\infty$ by $\varphi_{\mathrm{A}}^{j}$, that is $\varphi_{\mathrm{A}}^{j}=\varphi_{\mathrm{A}} \circ \varphi_{\mathrm{A}} \circ \cdots \circ \varphi_{\mathrm{A}}$. In addition, For a row contraction $\mathrm{A}=\left\{E_{k}\right\}_{k=1}^{n}$, we say that the operator sequence $\mathrm{A}$ is unital if $\sum_{k=1}^{n} E_{k} E_{k}^{*}=I$ is commutative, if $E_{k} E_{j}=E_{j} E_{k}$ for all $1 \leq k, j \leq n$ is normal, if each $E_{k}$ is normal and positive, and if every $E_{k}$ is positive. If $\mathrm{A}$ is unital (resp. commutative) then we say that $\varphi_{\mathrm{A}}$ is unital (resp. commutative). Moreover, $\varphi_{\mathrm{A}}$ or $\mathrm{A}$ is called trace preserving if $\sum_{k=1}^{n} E_{k}^{*} E_{k} \leq I$. For a subset $S \subset B(H)$, we denote the commutant of $S$ in $B(H)$ by $S^{\prime}$. We say that an $X \in B(H)$ is a fixed point of $\varphi_{\mathrm{A}}$ or a fixed point associated to the row contraction $\mathrm{A}$ if $\varphi_{\mathrm{A}}(X)=X$, Let $B(H)^{\varphi_{A}}$ be the set of fixed points of $\varphi_{\mathrm{A}}$. Some authors compared the commutant $\left\{E_{k, p} E_{k}^{*}, 1 \leq k \leq n\right\}^{\prime}$ of $\mathrm{A} \cup \mathrm{A}^{*}$, where $\mathrm{A}^{*}=\left\{E_{k}^{*}\right\}_{k=1}^{n}$, and some conditions for which $B(H)^{\varphi_{A}}=\left\{E_{k}, E_{k}^{*}, 1 \leq k \leq n\right\}$ are given (as in [1], [10]).

For a trace preserving quantum operation $\varphi_{\mathrm{A}}$, it was proved that $B(H)^{\varphi_{\mathrm{A}}}=\left\{E_{k}, E_{k}^{*}, 1 \leq k \leq n\right\}^{\prime}$ if $\operatorname{dim} H<\infty$ in [1]. And $B(H)^{\varphi_{A}}=\mathrm{A}^{\prime}$, if Kraus operators $\mathrm{A}$ is a spherical unitary [10]. On the other hand, the authors [12] consider some conditions for a unital and commuting row contraction $A$ to be normal and therefore $B(H)^{\varphi_{A}}=A^{\prime}$ in those cases. Moreover, the fixed points set $B(H)^{\varphi_{A}}$ of $\varphi_{\mathrm{A}}$ is represented when $\mathrm{A}$ is a commuting and trace preserving row contraction [15].

The purpose of this paper is to investigate fixed points of $j$-power of the completely positive map $\varphi_{\mathrm{A}}^{j}$ for $j \geq 1$. It is obtained that $B(H)^{\varphi_{A}^{2 j}}=B(H)^{\varphi_{A}}+B(H)^{-\varphi_{A}}$ and $B(H)^{\varphi_{A}^{2 j-1}}=B(H)^{\varphi_{A}}$ when A is self-adjoint and commutable. Furthermore, $B(H)^{\varphi_{A}^{j}}=B(H)^{\varphi_{A}}$ holds under certain condition.

\section{Main Results}

In this section, let A be a normal and commuting row contraction. To give main results, we begin with some notations and lemmas. Let $Q_{\mathrm{A}}=$ s.o. $-\lim _{j \rightarrow \infty} \varphi_{\mathrm{A}}^{j}(I)$ be the strong operator topology limit of $\left\{\varphi_{\mathrm{A}}^{j}(I)\right\}_{j=1}^{\infty}$.

Lemma 1 ([10]) Let $A=\left\{E_{k}\right\}_{k=1}^{n}$ be a unital and normal commuting row contractions. Then $B(H)^{\varphi_{A}}=A^{\prime}$.

Lemma 2 ([10]) Let $\mathrm{A}=\left\{E_{k}\right\}_{k=1}^{n} \subset B(H)$ be a commuting row contraction. If $Q_{\mathrm{A}} \neq 0$, then there exists a triple $\left\{K, \Gamma,\left\{U_{k}\right\}_{k=1}^{n}\right\}$ where $K$ is a Hilbert space, $\Gamma$ is a bounded operator from $K$ to $H$ and $\left\{U_{k}\right\}_{k=1}^{n}$ is a spherical unitary on $K$ satisfying the following properties:

1) $\Gamma \Gamma^{*}=Q_{\mathrm{A}}$;

2) $E_{k} \Gamma=\Gamma U_{k}$ for all $k$;

3) $K$ is the smallest reducing subspace for $\left\{U_{k}\right\}_{k=1}^{n}$ containing $\Gamma^{*} H$;

4) The mapping

$$
\rho:\left\{U_{k}\right\}_{k=1}^{n} \rightarrow B(H)^{\varphi_{A}}
$$

defined by

$$
\rho(Y)=\Gamma Y \Gamma^{*}, \quad Y \in\left\{U_{k}\right\}_{k=1}^{n}
$$

is a complete isometry from the commutant of $\left\{U_{k}\right\}_{k=1}^{n}$ onto the space $B(H)^{\varphi_{A}}$;

5) There exists a *-homomorphism $\pi: C^{*}\left\{I_{H}, B(H)^{\varphi_{A}}\right\} \rightarrow\left\{U_{k}\right\}_{k=1}^{n}$ such that

$$
\pi(\rho(Y))=Y, Y \in\left\{U_{k}\right\}_{k=1}^{n} .
$$


Lemma 3 ([16]) (Fuglede-Putnam Theorem) Let $A, B, C \in B(H)$, if $A$ and $B$ are normal, then $A C=C B$ implies $A^{*} C=C B^{*}$.

In general, there is no concrete relation between $B(H)^{\varphi_{\AA}^{k}}$ and $B(H)^{\varphi_{A}^{j}}$ for different positive integers $k$ and j.

Example 4 Let $E=\left(\begin{array}{cc}\frac{1}{2}+\frac{\sqrt{3}}{2} i & 0 \\ 0 & \frac{1}{2}-\frac{\sqrt{3}}{2} i\end{array}\right)$ and $A=\{E\}$, then $\varphi_{\mathrm{A}}(X)=E X E^{*}$ is well defined and

$B(H)^{\varphi_{A}}=\left\{\left(\begin{array}{cc}a_{11} & 0 \\ 0 & a_{22}\end{array}\right): a_{11}, a_{22} \in C\right\}$. However, by a direct computation, $\varphi_{\mathrm{A}}^{3}(X)=X$ and $B(H)^{\varphi_{\mathrm{A}}^{3}}=M_{2}(C)$.

Hence, $B(H)^{\varphi_{A}^{3}} \neq B(H)^{\varphi_{A}}$.

But if $\mathrm{A}$ is self-adjoint and commutable, the following result holds.

Theorem 5 If A is unital, self-adjoint and commutable, then $B(H)^{\varphi_{A}^{2 j-1}}=B(H)^{\varphi_{A}}$ and $B(H)^{\varphi_{A}^{2 j}}=B(H)^{\varphi_{A}}+B(H)^{-\varphi_{A}}$ for any $j \geq 1$.

Proof. For any $j \geq 1$, we first prove $B(H)^{\varphi_{A}^{j-1}}=B(H)^{\varphi_{A}}$. For any $X \in B(H)^{\varphi_{A}}$, then $\varphi_{A}(X)=X$. So $\varphi_{\mathrm{A}}^{j}(X)=\varphi_{\mathrm{A}}^{j-1}(X)=\cdots=X$ for any $j$. It is only to prove $B(H)^{\varphi_{\mathrm{A}}^{2 j-1}} \subset B(H)^{\varphi_{A}}$. According to A is unital, selfadjoint and commutable, then $\mathrm{A}=\left\{E_{k_{1}} E_{k_{2}} \cdots E_{k_{j}}: k_{i} \in \mathrm{A}\right.$ for $\left.1 \leq i \leq j\right\}$ is so. For any operator $A \in B(H)^{\varphi_{A}^{2 j-1}}$, then $A$ and $E_{k}^{2 j-1}$ are commutable for any $k$ by lemma 1 . By the function calculus, $A$ and $E_{k}$ are commutable since $E_{k}$ is self-adjoint, and so $A \in B(H)^{\varphi_{A}}$. Therefore,

$$
B(H)^{\varphi_{A}^{2 j-1}}=B(H)^{\varphi_{A}} .
$$

Next, we prove $B(H)^{\varphi_{A}^{2 j}}=B(H)^{\varphi_{A}}+B(H)^{-\varphi_{A}}$. For any $A \in B(H)^{\varphi_{A}^{2 j}}$, then $A E_{k} E_{k_{1}}^{2} \cdots E_{k_{j-1}}^{2} E_{l}=E_{k} E_{k_{1}}^{2} \cdots E_{k_{j-1}}^{2} E_{l} A$, for any $k, k_{1}, k_{2}, \cdots, k_{j-1}, l \in\{1,2, \cdots, n\}$.

So $A E_{k} E_{l}=E_{k} E_{l} A$ since $\sum_{k=1}^{n} E_{k}^{2}=I$, thus $A \in B(H)^{\varphi_{A}^{2}}$. It follows that $\frac{1}{2}\left(A+\varphi_{\mathrm{A}}(A)\right) \in B(H)^{\varphi_{A}}$ and $\frac{1}{2}\left(A-\varphi_{\mathrm{A}}(A)\right) \in B(H)^{-\varphi_{A}}$. So $B(H)^{\varphi_{A}^{2 j}} \subset B(H)^{\varphi_{A}}+B(H)^{-\varphi_{A}}$. Conversely, for any $X \in B(H)^{\varphi_{A}}$, $Y \in B(H)^{-\varphi_{\mathrm{A}}}$, then $\varphi_{\mathrm{A}}(Y)=-Y$. So $\varphi_{\mathrm{A}}^{2 j}(Y)=\varphi_{\mathrm{A}}^{2 j-1}(-Y)=\varphi_{\mathrm{A}}^{2 j-2}(Y)=\cdots=Y$ for any $j$. Therefore $\varphi_{\mathrm{A}}^{2 j}(X+Y)=\varphi_{\mathrm{A}}^{2 j-1}(X-Y)=\varphi_{\mathrm{A}}^{2 j-2}(X+Y)=\cdots=Y$. So $B(H)^{\varphi_{\mathrm{A}}}+B(H)^{-\varphi_{\mathrm{A}}} \subset B(H)^{\varphi_{\mathrm{A}}^{2 j}}$ and $B(H)^{\varphi_{A}}+B(H)^{-\varphi_{A}}=B(H)^{\varphi_{A}^{2 j}}$. Therefore, the result holds. This completes the proof.

Corollary 6 Let $\mathrm{A}=\left\{E_{k}\right\}_{k=1}^{n}$ be unital, self-adjoint and commutable, then

$$
\mathrm{A} \cdot \mathrm{A}=\mathrm{A}^{\prime}+\operatorname{Iw}(\mathrm{A},-\mathrm{A}) \text {, }
$$

where $I w(\mathrm{~A},-\mathrm{A})=\left\{X \in B(H): E_{k} X+X E_{k}=0\right.$, for any $\left.k\right\}$.

Proof. From Theorem 5 and Lemma 1, it is only to prove that $B(H)^{-\varphi_{A}}=I w(\mathrm{~A},-\mathrm{A})$. Let $F_{k}=\left(\begin{array}{cc}E_{k} & 0 \\ 0 & -E_{k}\end{array}\right)$ and $\mathrm{B}=\left\{F_{k}\right\}_{k=1}^{n}$, for any operator $X \in B(H)^{-\varphi_{A}}$, then $\left(\begin{array}{cc}0 & X \\ 0 & 0\end{array}\right) \in B(H \oplus H)^{\varphi_{\mathrm{B}}}$.

From Lemma 1, we have

$$
\left(\begin{array}{cc}
0 & X \\
0 & 0
\end{array}\right)\left(\begin{array}{cc}
E_{k} & 0 \\
0 & -E_{k}
\end{array}\right)=\left(\begin{array}{cc}
E_{k} & 0 \\
0 & -E_{k}
\end{array}\right)\left(\begin{array}{cc}
0 & X \\
0 & 0
\end{array}\right)
$$


It follows that $E_{k} X+X E_{k}=0$ for any $k$ and then $X \in I w(\mathrm{~A},-\mathrm{A})$. This completes the proof.

Theorem 7 Let $\mathrm{A}=\left\{E_{k}\right\}_{k=1}^{n}$ be unital and commutable. Supposing that there is an $k_{0}$ such that $E_{k_{0}} \in \mathrm{A}$ is positive and invertible, then $B(H)^{\varphi_{A}^{j}}=B(H)^{\varphi_{A}}$, where $1 \leq j<\infty$.

Proof. From Lemma 2, there exists a triple $\left\{K, \Gamma,\left\{U_{k}\right\}_{k=1}^{n}\right\}$ where $K$ is a Hilbert space, $\Gamma: K \rightarrow H$ is abounded operator and $\left\{U_{k}\right\}_{k=1}^{n}$ is a normal unital and commuting operator sequence on $K$ having the properties 1) $\Gamma \Gamma^{*}=I$; 2) $E_{k} \Gamma=\Gamma U_{k}$; 3) $K$ is the smallest reducing subspace for $\left\{U_{k}\right\}_{k=1}^{n}$ containing $\Gamma^{*} H$; 4) The mapping $\rho:\left\{U_{k}\right\}_{k=1}^{n} \rightarrow B(H)^{\varphi_{A}}$ defined by $\rho(Y)=\Gamma Y \Gamma^{*}$ and $Y \in\left\{U_{k}\right\}_{k=1}^{n '}$ is a complete isometry from the commutant of $\left\{U_{k}\right\}_{k=1}^{n}$ onto the space $B(H)^{\varphi_{\mathrm{A}}}$; also it is obtained that $U_{k}=\pi\left(E_{k}\right)$ for any $k$, where $\pi: C^{*}\left\{I_{H}, B(H)^{\varphi_{A}}\right\} \rightarrow B(K)$ is a unital *-homomorphism. Then $U_{k_{0}}=\pi\left(E_{k_{0}}\right)$ is positive and invertible since $E_{k_{0}}$ is positive and invertible. Next, we write $\Lambda=\left\{\left\{k_{1}, k_{2}, \cdots, k_{j}\right\}: 1 \leq k_{1}, k_{2}, \cdots, k_{j} \leq n\right\}$ and $U_{\alpha}=U_{k_{1}} U_{k_{2}} \cdots U_{k_{j}}$ for any $\alpha=\left\{k_{1}, k_{2}, \cdots, k_{j}\right\} \in \Lambda$. In fact, $U_{\alpha} A=A U_{\alpha}$ if and only if $U_{k} A=A U_{k}$ for any $1 \leq k \leq n$. On one hand, $U_{\alpha} A=A U_{\alpha}$ if $U_{k} A=A U_{k}$; on the other hand, if $U_{\alpha} A=A U_{\alpha}$, then $U_{k_{0}}^{j} A=A U_{k_{0}}^{j}$ and so $U_{k_{0}} A=A U_{k_{0}}$ by the function calculus. Moreover, $A U_{k_{0}}^{j-1} U_{k}=U_{k_{0}}^{j-1} U_{k} A=U_{k_{0}}^{j-1} A U_{k}$, thus $U_{k} A=A U_{k}$ since $U_{k_{0}}^{j-1}$ is invertible. That is to say, $\left\{U_{\alpha}\right\}_{\alpha \in \Lambda}$ and $\left\{U_{k}\right\}_{k=1}^{n}$ have the same reducing subspace. It follows that $K$ is also the smallest reducing subspace for the unital, normal and commuting operator sequence $\left\{U_{\alpha}\right\}_{\alpha \in \Lambda}$ containing $\Gamma^{*} H$. Thus $\rho(Y)=\Gamma Y \Gamma^{*}, Y \in\left\{U_{\alpha}\right\}_{\alpha \in \Lambda}{ }^{\prime}$ is also a complete isometry from the commutant of $\left\{U_{\alpha}\right\}_{\alpha \in \Lambda}$ onto the space $(H)^{\varphi_{A}^{j}}$. Combining with $B(H)^{\varphi_{A}} \subset B(H)^{\varphi_{A}^{j}}$, it is easy to get $B(H)^{\varphi_{A}^{j}}=B(H)^{\varphi_{A}}$. The proof is completed.

\section{Acknowledgements}

This research was supported by the Natural Science Basic Research Plan of Henan Province (No. 14 B110010 and No. 1523000410221).

\section{References}

[1] Arias, A., Gheondea, A. and Gudder, S. (2002) Fixed Points of Quantum Operations. Journal of Mathematical Physics, 43, 5872-5881. http://dx.doi.org/10.1063/1.1519669

[2] Li, Y. (2011) Characterizations of Fixed Points of Quantum Operations. Journal of Mathematical Physics, 52, Article ID: 052103. http://dx.doi.org/10.1063/1.3583541

[3] Long, L. and Zhang, S.F. (2011) Fixed Points of Commutative Super-Operators. Journal of Physics A: Mathematical and Theoretical, 44, Article ID: 09521. http://dx.doi.org/10.1088/1751-8113/44/9/095201

[4] Long, L. (2011) A Class of General Quantum Operations. International Journal of Theoretical Physics, 50, $1319-1324$. http://dx.doi.org/10.1007/s10773-010-0627-4

[5] Liu, W.H. and Wu, J.D. (2010) Fixed Points of Commutative Lüders Operations. Journal of Physics A: Mathematical and Theoretical, 43, Article ID: 395206. http://dx.doi.org/10.1088/1751-8113/43/39/395206

[6] Magajna, B. (2012) Fixed Points of Normal Completely Positive Maps on B(H). Journal of Mathematical Analysis and Applications, 389, 1291-1302. http://dx.doi.org/10.1016/j.jmaa.2012.01.007

[7] Nagy, G. (2008) On Spectra of Lüders Operations. Journal of Mathematical Physics, 49, Article ID: 022110. http://dx.doi.org/10.1063/1.2840472

[8] Popescu, G. (2003) Similarity and Ergodic Theory of Positive Linear maps. Journal für die reine und angewandte Mathematik, 561, 87-129. http://dx.doi.org/10.1515/crll.2003.069 
[9] Prunaru, B. (2008) Toeplitz Operators Associated to Commuting Row Contractions. Journal of Functional Analysis, 254, 1626-1641. http://dx.doi.org/10.1016/j.jfa.2007.11.001

[10] Prunaru, B. (2011) Fixed Points for Lüders Operations and Commutators. Journal of Physics A: Mathematical and Theoretical, 44, Article ID: 185203. http://dx.doi.org/10.1088/1751-8113/44/18/185203

[11] Zhang, H.Y. and Ji, G.X. (2012) A Note on Fixed Points of General Quantum Operations. Reports on Mathematical Physics, 70, 111-117. http://dx.doi.org/10.1016/S0034-4877(13)60016-6

[12] Zhang, H.Y. and Ji, G.X. (2013) Normality and Fixed Points Associated to Commutative Row Contractions. Journal of Mathematical Analysis and Applications, 400, 247-253. http://dx.doi.org/10.1016/j.jmaa.2012.10.042

[13] Choi, M.D. (1975) Completely Positive Linear Maps on Complex Matrices. Linear Algebra and Its Applications, 10, 285-290. http://dx.doi.org/10.1016/0024-3795(75)90075-0

[14] Kraus, K. (1971) General State Changes in Quantum Theory. Annals of Physics, 64, 311-355. http://dx.doi.org/10.1016/0003-4916(71)90108-4

[15] Zhang, H.Y. and Xue, M.Z. (2016) Fixed Points of Trace Preserving Completely Positive maps. Linear Multilinear A, 64, 404-411. http://dx.doi.org/10.1080/03081087.2015.1043718

[16] Hou, J.C. (1985) On Putnam-Fuglede Theorem of Nonnormal Operators. Acta Mathematica Sinica, 28, 333-340. 